\title{
Termorretificação do alburno e cerne da madeira de Auxemma oncocalyx
}

\author{
Tallyta Martins de Sousa ${ }^{1}$ Vinicius Gomes Castro@ ${ }^{1 *}$
}

${ }^{1}$ Universidade Federal Rural do Semiárido, Av. Francisco Mota, 572, Pres. Costa e Silva, Mossoró, RN, Brasil

\section{Original Article \\ *Corresponding author: vinicius.castro@ufersa.edu.b $\mathrm{r}$ \\ Palavras-chave: \\ Caatinga}

Pau Branco

Colorimetria

Keywords:

Caatinga

Pau Branco

Colorimetry

Received in

2021/06/30

Accepted on

2021/05/10

Published in

2021/10/11

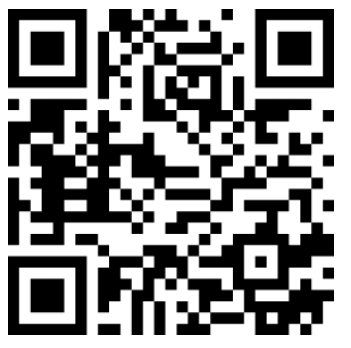

DOI:

https://doi.org/10.34062/afs. v8i3.12698

\section{(cc) BY}

RESUMO: O objetivo deste estudo foi a análise dos efeitos da termorretificação na madeira de alburno de Auxemma oncocalyx, utilizando duas temperaturas distintas, em busca de se obter homogeneidade nas propriedades físico-mecânicas e cor entre as partes do cerne e alburno. As propriedades físico-mecânicas avaliadas foram massa específica básica, luminosidade da cor, estabilidade dimensional, resistência à compressão paralela às fibras e módulo de elasticidade. Os resultados indicaram que o ganho na massa específica da madeira modificada ocorre somente em temperatura mais baixas, quando a perda de massa pela degradação dos componentes fundamentais da madeira foi menor do que a redução no volume do material. O efeito do tratamento térmico na higroscopicidade da madeira também gerou peças de alburno dimensionalmente mais estáveis. Em ambas as temperaturas testadas, a resistência à compressão foi superior em madeiras de alburno termorretificada comparadas com as madeiras de alburno e cerne não tratados. Com base nestes resultados, foi possível recomendar o uso de termorretificação da madeira de alburno de Pau-branco para que se produza produtos de madeira com aparência e características tecnológicas semelhantes aos que usam madeira do cerne desta espécie, aumentando assim o rendimento de produtos de qualidade.

\section{Thermo modification of Auxemma oncocalyx sapwood and heartwood}

\begin{abstract}
The aim of this work was to analyse the effect of thermally modification of Auxemma oncocalyx (Pau-branco) sapwood, using two different temperatures, to obtain homogeneity between physico-mechanical properties and color of sapwood and heartwood. The physico-mechanical properties evaluated were basic density, luminosity of the color, dimensional stability, parallel compression strength and modulus of elasticity. The results showed increase of density of sapwood thermally treated only with lower temperatures applied, when the weight loss due structural components of wood degradation was lower than volume shrinkage. The effect of thermal treatment on the wood hygroscopicity also resulted in more dimensional stable sapwood samples. For both tested temperatures, the compression strength was higher for thermally modified sapwood than non-treated sapwood or heartwood. Based on those results, it is possible to indicate the use of thermally modified treatment to Pau-branco sapwood for production of wood products with similar look and technologic characteristics of the ones that use the heartwood of this species, increasing the yield of high-quality products.
\end{abstract}




\section{Introdução}

A Caatinga é a maior Floresta Tropical Sazonalmente Seca (FTSS) do mundo, com uma área de $912.529 \mathrm{~km}^{2}$. Contudo, este bioma vem sofrendo uma pressão crescente sobre seus recursos naturais, chegando ao ponto de $35,95 \%$ da área encontrar-se em processo de desertificação (Silva et al. 2018). Uma das principais razões desta devastação é a exploração madeireira ilegal e não sustentável para suprir o consumo de lenha nativa para fins domésticos e industriais (Kill e Porto, 2019). Em muitos casos, a lenha é a única alternativa de fonte de energia para comunidades rurais de baixo poder aquisitivo que não conseguem pagar pelo gás de cozinha (Lima et al. 2018).

Uma alternativa para reduzir a pressão, seria uma exploração mais sustentável, sob plano de manejo, que pudesse gerar renda para a comunidade local sem devastar a Caatinga remanescente. Desenvolver planos de manejo é responsabilidade dos órgãos ambientais estaduais, por exemplo o Instituto de Desenvolvimento Sustentável e Meio Ambiente (IDEMA) no Rio Grande do Norte, mas para que seja possível, é necessário estudar e compreender o verdadeiro potencial madeireiro da região e oferecer produtos com o maior valor agregado possível.

Auxemma oncocalyx, (Allemão) Taub., conhecida popularmente como Pau-branco, é uma árvore da família Boraginaceae nativa da Caatinga que pode chegar a uma densidade de 4200 indivíduos/ha (Ivanov et al. 2019), abundante principalmente nos estados do Ceará e Rio Grande do Norte (Melo et al. 2018). É espécie secundária tardia de crescimento lento, com um incremento médio anual de $1,83 \mathrm{~mm} / \mathrm{ano}$ (Calvo-Rodrigues et al. 2017). A madeira do Pau-branco é considerada moderadamente pesada e com uma boa resistência mecânica, adequada, como por exemplo, na fabricação de painéis colados lateralmente (Souza et al. 2020). Porém uma de suas principais características físicas da madeira do Pau-branco é a presença de cerne e alburno com aparência e propriedades bem distintas. Na prática, apenas a madeira do cerne é desejada, o que gera um problema durante o processo de industrialização reduzindo o rendimento e aumentando a geração de um resíduo com grande potencial de aproveitamento.

A técnica de termorretificação vem sendo aplicada para diversos fins e pode ser uma possível solução para a dualidade da madeira do Pau-branco. Termorretificação é um método que consiste em submeter peças de madeira a temperaturas entre 120 e $200^{\circ} \mathrm{C}$, mais elevadas do que as utilizadas no processo de secagem, sem que haja a carbonização. Esse processo degrada as hemiceluloses e condensa a lignina e celulose presentes na madeira, e como resultado pode vir a melhorar suas propriedades mecânicas (Modes et al. 2017). O controle da temperatura e tempo de exposição também afeta a aparência da madeira, uma vez que as hemiceluloses e celuloses refletem a luz visível. Desta forma, o processo de termo degradação destes componentes afeta a luminosidade das peças (Schulz et al. 2021).

Diante do exposto, o objetivo deste trabalho foi a análise dos efeitos da termorretificação na madeira de alburno de Auxemma oncocalyx, utilizando duas temperaturas distintas, em busca de se obter homogeneidade nas propriedades físicas, mecânicas e cor entre as partes do cerne e alburno.

\section{Material e Métodos}

As amostras utilizadas foram obtidas de madeiras de cerne e alburno retiradas a altura do peito de três indivíduos de Auxemma oncocalyx (Pau-branco) com diâmetro à altura do peito (DAP) de aproximadamente $20 \mathrm{~cm}$, coletados em uma propriedade manejada, na zona rural do município de Mossoró, Rio Grande do Norte.

Corpos de prova produzidos a partir de madeiras de alburno foram submetidos ao processo de termorretificação em duas temperaturas. As amostras foram colocadas na estufa ainda em processo de aquecimento, programada para atingir inicialmente a temperatura de $140^{\circ} \mathrm{C}$ por 1 hora. Decorrido este tempo, foram retirados os corpos de prova então denominados como tratamento T140. Os demais corpos de prova foram mantidos na estufa que teve uma nova temperatura de $200^{\circ} \mathrm{C}$ reprogramada. Uma vez a nova temperatura alvo foi atingida, as amostras agora denominadas T200 permaneceram por mais 1 hora (Figura 1). 


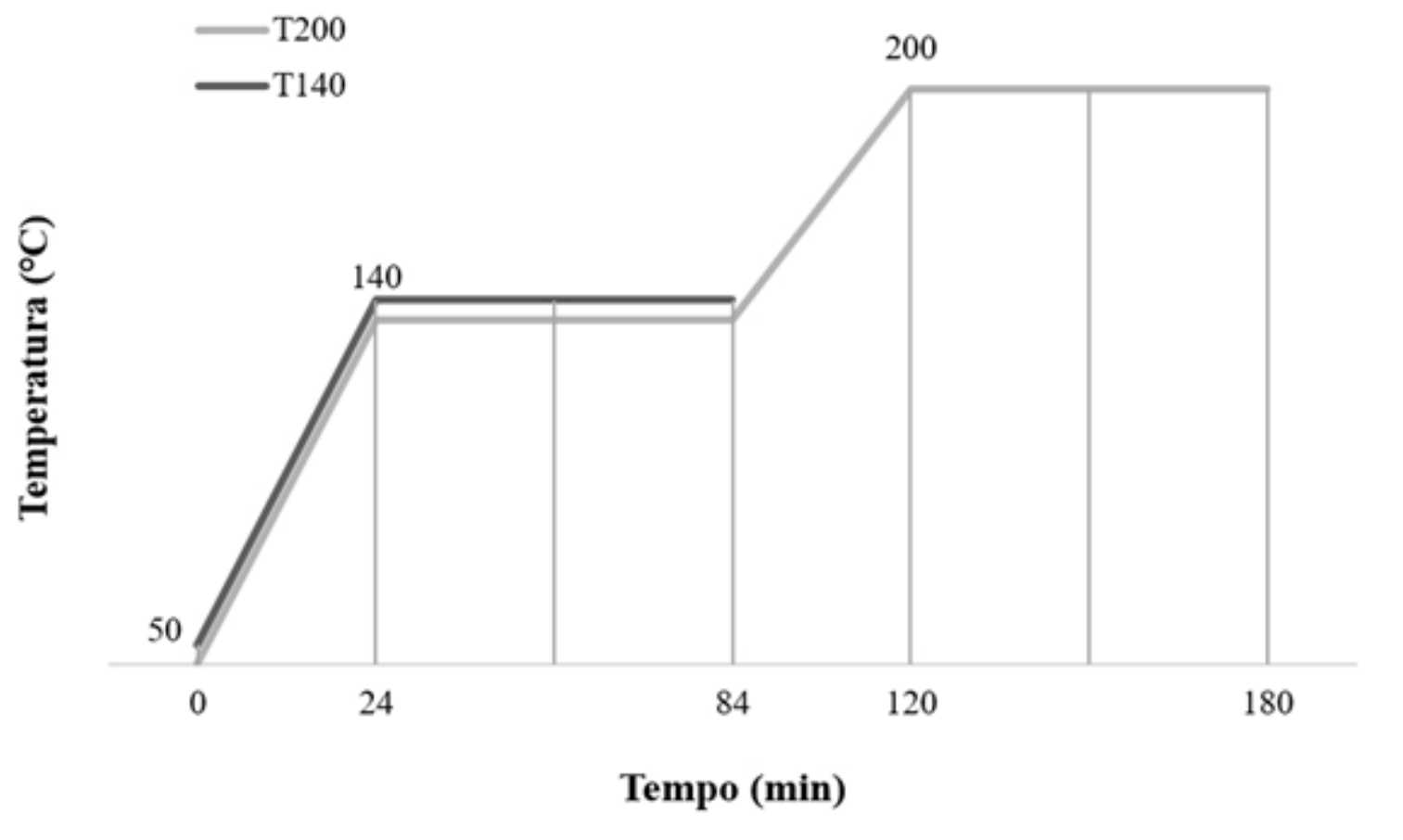

Figura 1. Rampa de aquecimento para os tratamentos térmicos a $140^{\circ} \mathrm{C}(\mathrm{T} 140)$ e $200^{\circ} \mathrm{C}(\mathrm{T} 200)$ aplicados à madeira de alburno de Pau-branco.

Para as madeiras de cerne e alburno nãotratadas e amostras de alburno temorretificadas T140 e T200, foram determinadas a massa específica básica, índices de contração lineares (tangencial, radial e longitudinal), volumétrico, coeficiente de anisotropia, resistência à compressão paralela e módulo de elasticidade $\mathrm{E}_{\mathrm{w} 0}$ na direção paralela às fibras (ABNT 1997). Para as propriedades físicas, foram amostrados 74 corpos de prova com as dimensões $2 \times 2 \times 5 \mathrm{~cm}$, no sentido tangencial, radial e longitudinal respectivamente (Tabela 1). Para a propriedade mecânica, foram testados 16 corpos de prova de $3 \times 3 \times 9 \mathrm{~cm}$, sendo 4 para cada tipo de tratamento.

Tabela 1. Delineamento experimental para análise das propriedades físicas.

\begin{tabular}{cc}
\hline Amostra & $\begin{array}{c}\text { Número de corpos de } \\
\text { prova }\end{array}$ \\
\hline Cerne não tratado & 18 \\
Alburno não tratado & 18 \\
T140 & 19 \\
T200 & 19 \\
\hline
\end{tabular}

Em que: $\mathrm{T} 140=$ alburno termorretificado a $140^{\circ} \mathrm{C}$; $\mathrm{T} 200=$ alburno termorretificado a $200^{\circ} \mathrm{C}$.

Para determinar o efeito dos tratamentos na luminosidade e consequentemente na cor das madeiras de alburno termorretificadas, foram medidos três pontos em cada cinco amostras de alburno e cerne não tratados e do alburno T140 e T200 utilizando colorímetro Konica Minolta CR410 que mensura conforme o sistema CIE-L*a*b*. Antes da medição do parâmetro colorimétrico L (luminosidade), as amostras foram lixadas manualmente seguindo a ordem de lixas de grãos 80 , 150 e 220

A análise estatística dos dados foi baseada na realização de análise de variância (ANOVA) para as avaliações das propriedades físicas e mecânicas da madeira de cerne e alburno sem e com tratamento a 140 e $200^{\circ} \mathrm{C}$. Foi aplicado teste de diferenciação de médias Tukey a 5\% de significância.

\section{Resultados}

A madeira de cerne teve seu valores médio de massa específica básica entre 0,76 e $0,95 \mathrm{~g} / \mathrm{cm}^{3}$, que de acordo com Zaque et al. (2019) podem ser classificadas como madeiras pesadas (Tabela 2). Já as madeiras de alburno com ou sem tratamento térmico possuem valores mais baixos de massa específica básica que as classificam como moderadamente pesada (valores entre 0,56 e 0,75 $\left.\mathrm{g} / \mathrm{cm}^{3}\right)$. 
Sousa et al.

Tabela 2. Valores médios para as propriedades físicas de cerne, alburno e alburno termorretificado de Auxemma oncocalyx (Pau-branco).

\begin{tabular}{ccccc}
\hline Propriedades físicas & Cerne & Alburno & T140 & T200 \\
\hline Massa Específica Básica $\left(\mathrm{g} / \mathrm{cm}^{3}\right)$ & $0,77 \mathrm{a}$ & $0,67 \mathrm{c}$ & $0,72 \mathrm{ab}$ & $0,68 \mathrm{bc}$ \\
& $(0,05)$ & $(0,08)$ & $(0,10)$ & $(0,13)$ \\
$\beta$ V max (\%) & $22,57 \mathrm{~b}$ & $30,08 \mathrm{a}$ & $25,65 \mathrm{~b}$ & $25,12 \mathrm{~b}$ \\
& $(0,17)$ & $(0,13)$ & $(0,10)$ & $(0,19)$ \\
$\beta$ linear T (\%) & $5,44 \mathrm{~b}$ & $5,89 \mathrm{ab}$ & $6,48 \mathrm{a}$ & $3,78 \mathrm{c}$ \\
& $(0,13)$ & $(0,09)$ & $(0,14)$ & $(0,11)$ \\
$\beta$ linear R (\%) & $3,71 \mathrm{ab}$ & $4,32 \mathrm{a}$ & $4,34 \mathrm{a}$ & $3,14 \mathrm{~b}$ \\
& $(0,18)$ & $(0,19)$ & $(0,13)$ & $(0,22)$ \\
$\beta$ linear L (\%) & $0,28 \mathrm{a}$ & $0,21 \mathrm{a}$ & $0,18 \mathrm{a}$ & $0,17 \mathrm{a}$ \\
& $(0,74)$ & $(0,81)$ & $(0,76)$ & $(0,81)$ \\
Coeficiente de anisotropia & $1,54 \mathrm{a}$ & $1,45 \mathrm{a}$ & $1,54 \mathrm{a}$ & $1,33 \mathrm{a}$ \\
& $(0,19)$ & $(0,22)$ & $(0,17)$ & $(0,31)$ \\
Luminosidade & $29,30 \mathrm{~b}$ & $55,45 \mathrm{a}$ & $60,27 \mathrm{a}$ & $26,33 \mathrm{~b}$ \\
& $(0,02)$ & $(0,33)$ & $(0,02)$ & $(0,02)$
\end{tabular}

Em que: $\mathrm{T} 140=$ alburno termorretificado a $140^{\circ} \mathrm{C} ; \mathrm{T} 200=$ alburno termorretificado a $200^{\circ} \mathrm{C} ; \beta \mathrm{V}$ max $=$ contração volumétrica máxima; $\beta$ linear $\mathrm{T}=$ contração linear tangencial; $\beta$ linear $\mathrm{R}=$ contração linear radial; $\beta$ linear $\mathrm{L}=$ contração linear longitudinal; Valores seguidos de mesma letra em uma mesma linha não diferem estatisticamente entre si no teste de Tukey a 5\% de significância; Valores entre parênteses referentes ao coeficiente de variação.

Embora possa ser observado que o cerne é mais estável do que o alburno, e que o tratamento térmico igualou estatisticamente os valores de contração volumétricos, a madeira do Pau-branco foi considerada dimensionalmente instável como um todo. Eleotério et al. (2015) definiram o limite de contração volumétrico de 19,40\%, madeiras que apresentam valores médios superiores a este podem ser classificadas como altamente instáveis.

A classificação em relação as contrações lineares foram divergentes da volumétrica. A contração radial do cerne e alburno ficou dentro do limite de 3,52 a 5,59\% considerado como de média estabilidade (Eleotério et al. 2015). Porém, após tratamento a $200^{\circ} \mathrm{C}$, a madeira termorretificada de alburno passou a ser classificada como de baixa contração.

A contração tangencial foi classificada como baixa, uma vez que todos os valores foram abaixo do limite de 7,43\% (Eleotério et al. 2015). Em relação a contração longitudinal, os valores médios de todos os tratamentos não diferiram estatisticamente e se mantiveram dentro da média da maioria das madeiras que é entre 0,1 e 0,2\% (Glass e Zelinka, 2010), que as torna apta para usos estruturais.

O coeficiente de anisotropia indica a propensão da madeira empenar durante o processo de secagem. Madeiras com valores médios abaixo de 1,5 são consideradas estáveis e de excelente qualidade (Klitzke et al. 2008). As madeiras de Paubranco encontraram-se no limiar desta classificação, uma vez que não houve diferença estatística entre os tratamentos.

Quanto a cor, o parâmetro luminosidade (L) é um valor que varia entre 0, para cores absolutamente pretas, e 100, para o branco absoluto, sendo madeiras com $\mathrm{L}>56$ podem ser classificadas como claras (Maia et al. 2020). A diferença estatística entre os valores médios do cerne e do alburno quantificam a diferença visual destas madeiras (Figura 1). O alburno apresentou um valor médio no limite da classificação como madeira clara, que originou o nome popular da espécie. Já o cerne apresentou valores abaixo de 30 , sendo uma madeira bem escura. Nota-se que a termorretificação a $200^{\circ} \mathrm{C}$ foi capaz de alterar a cor do alburno, escurecendo-o de tal forma que passou a ser estatisticamente igual ao do cerne. 


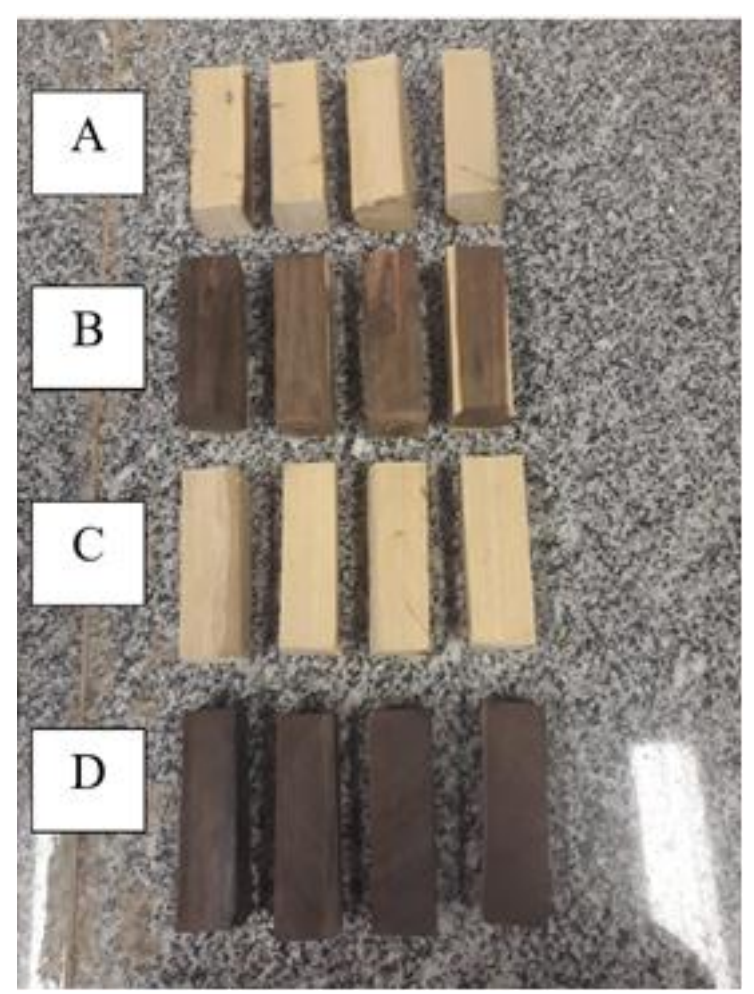

Figura 1. Aspecto visual das amostras de madeira de Auxemma oncocalyx (Pau-branco). Em que: A Alburno; B - Cerne; C - Alburno tratado a $140^{\circ} \mathrm{C}$, e D - Alburno tratado a $200^{\circ} \mathrm{C}$.

Em relação as propriedades mecânicas, foi possível observar dois comportamentos distintos. Para a compressão paralela às fibras, não houve diferença estatística entre os valores de cerne e alburno do Pau-branco, mas houve um aumento da resistência quando o alburno foi tratado independente da temperatura (Figura 2). Já para o módulo de elasticidade, não houve diferença estatística entre nenhum dos tratamentos (Figura 3). De acordo com a norma brasileira para estrutura de madeira (ABNT, 1997), o Pau-branco pode ser considerada como uma madeira da classe C30 (madeiras de folhosas com requisitos mínimos de resistência à compressão paralela às fibras de $30 \mathrm{MPa}$ e módulo de elasticidade $\mathrm{E}_{\mathrm{w} 0}$ de $14,5 \mathrm{GPa}$ ).

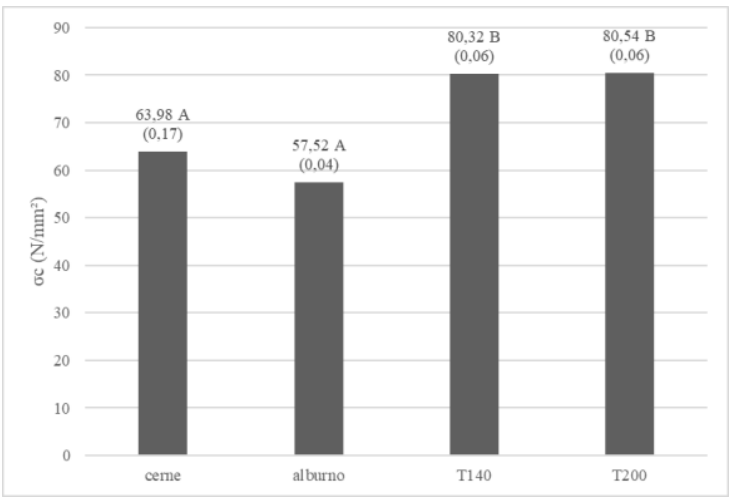

Figura 2. Valores de resistência à compressão paralela às fibras para a madeira de cerne e alburno tratado e não tratado termicamente a $140^{\circ} \mathrm{C}$ e $200^{\circ} \mathrm{C}$ de Auxemma oncocalyx (Pau-branco). Em que: valores seguidos por mesma letra não diferem estatisticamente; valores entre parênteses são referentes ao coeficiente de variação.

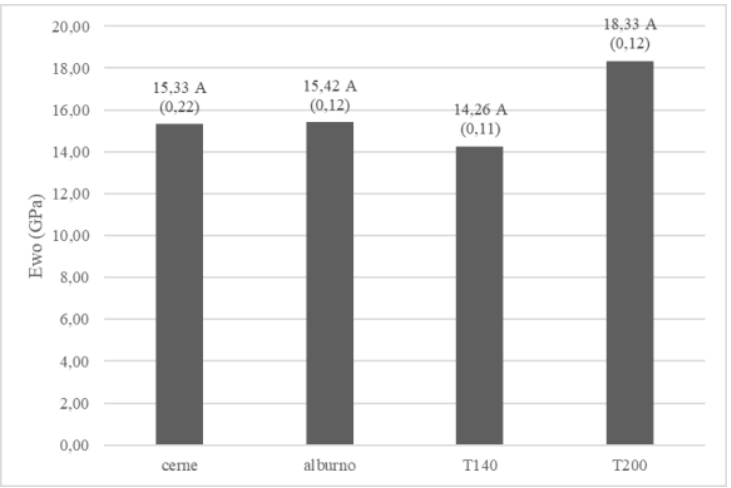

Figura 3. Valores de módulo de elasticidade $\mathrm{E}_{\mathrm{w} 0}$ na direção paralela às fibras para a madeira de cerne $\mathrm{e}$ alburno tratado e não tratado termicamente a $140^{\circ} \mathrm{C}$ e $200^{\circ} \mathrm{C}$ de Auxemma oncocalyx (Pau-branco). Em que: valores seguidos por mesma letra não diferem estatisticamente; valores entre parênteses são referentes ao coeficiente de variação.

\section{Discussões}

O Pau-branco, como diversas outras espécies, apresentou a madeira de cerne mais densificada do que a madeira do alburno. Isso ocorre porque o cerne tende a possuir mais extrativos no interior de suas células, além da possibilidade da presença de obstruções em seus vasos conhecidas como tilose, que visam impedir a passagem de umidade e consequentemente proteger o troco da árvore de ataques de agentes biodeterioradores após o processo de morte das células do alburno (Melo e Camargos, 2016).

$\mathrm{O}$ tratamento térmico a $140^{\circ} \mathrm{C}$ da madeira de alburno densificou a madeira a ponto de apresentar uma massa específica estatisticamente semelhante ao cerne. Porém, ao aumentar a temperatura para $200^{\circ} \mathrm{C}$, as amostras passaram a ter uma tendencia de reduzir a sua massa específica. Na literatura é possível encontrar diversos casos de madeiras que tem sua massa específica reduzida com tratamentos térmicos. Esse comportamento ocorre porque hemiceluloses degradadas e extrativos passam a serem volatilizados com a aplicação do calor, reduzindo o peso da madeira e consequentemente sua massa específica (Tuncer e Dogu, 2018).

Uma vez que o alburno apresenta menor quantidade de extrativos do que o cerne, a redução da massa específica não ocorre necessariamente. Neste caso, o que ocorre é a redução do volume saturado do material, que influencia no aumento da massa específica básica. $\mathrm{O}$ tratamento térmico pode afetar as hidroxilas livres presente na região amorfa 
da celulose (Bachle et al. 2010), reduzindo assim o processo de histerese, ou seja, a capacidade de absorção de água pela parede celular.

No caso deste trabalho, a temperatura de $140^{\circ} \mathrm{C}$ não foi o suficiente para volatilizar os componentes químicos do alburno, mas já reduziu a capacidade de absorção da celulose. Quando a temperatura aplicada foi de $200^{\circ} \mathrm{C}$, a evaporação do material volátil teve uma influência semelhante a da perda de volume, fazendo com que o valor da massa específica básica fosse estatisticamente igual ao do alburno não tratado. Esse padrão de comportamento também foi observado por Severo et al. (2012), que concluíram que a massa específica não seria a forma mais adequada de se avaliar a qualidade de uma madeira modificada.

Nogueira et al. (2019) afirmaram que pode haver uma correlação negativa entre contração volumétrica e massa específica em madeiras da Caatinga. Porém, neste estudo essa correlação poderia explicar apenas a diferença entre a estabilidade do cerne e alburno não tratados. Isso porque, a madeira de alburno tratado a $200^{\circ} \mathrm{C}$ apresenta uma massa específica estatisticamente igual ao do alburno não tratado, mas contrações lineares e volumétrica menores. A degradação térmica das celuloses amorfas e das hemiceluloses, ambas ricas em hidroxilas livres responsáveis pela capacidade higroscópica da madeira, também afetam a estabilidade dimensional (Severo et al. 2016).

Um dos efeitos mais conhecidos do tratamento térmico, e que foi observado neste trabalho em tratamento a $200^{\circ} \mathrm{C}$, é o escurecimento da madeira. A madeira tende a escurecer também por causa da degradação térmica das hemiceluloses e da celulose, que normalmente refletem a luz visível criando a percepção de cores claras (Schulz et al. 2021). As madeiras de alburno termorretificadas tendem a sofrer maiores alterações em suas cores do que as de cerne, sendo a razão associada as diferenças da composição química entre essas partes da madeira (Tuncer e Dogu, 2018). Além do efeito nos componentes fundamentais da parede celular, a migração dos extrativos durante o tratamento pode afetar a percepção da cor. Maia et al. (2020) apontaram uma correlação positiva entre a luminosidade e os teores de extrativos apolares.

O tratamento térmico do alburno nas temperaturas de 140 e $200^{\circ} \mathrm{C}$ foi capaz de melhorar a resistência à compressão das amostras, mas não chegaram a afetar a rigidez do material. Roszyk et al. (2020) também não acharam correlação entre a modificação térmica e módulo de elasticidade medido pela compressão longitudinal, mas apontaram que a compressão paralela às fibras é afetada pela temperatura usada no processo de termorretificação. Menezes et al. (2019) observaram resultados que indicaram que a $180^{\circ} \mathrm{C}$, duas espécies de Eucalipto (Corymbia citriodora e Eucalyptus saligna) termorretificados tiveram decréscimo nos valores tanto de módulo de elasticidade, quanto de compressão paralela.

O efeito do tratamento térmico nas propriedades mecânicas pode estar associado a dois fatores: 1 - Embora a termorretificação degrade em grande parte as hemiceluloses, os outros polímeros (celulose e lignina, responsáveis pela resistência e dureza da madeira) aumentam em proporção (Sikora et al. 2018); 2 - Propriedades mecânicas são conhecidamente afetadas negativamente pelo teor de umidade das peças. Como a modificação térmica afeta a capacidade da madeira de absorver água, consequentemente tende a melhorar suas resistências (Roszyk et al. 2020).

\section{Conclusões}

É possível alterar termicamente a madeira de alburno de Auxemma oncocalyx (Pau-branco) e torná-la esteticamente semelhante a madeira de cerne. A homogeneização das partes distintas do tronco da espécie não são somente visuais, uma vez que há também ganho de massa específica básica e resistência mecânica, visto no ensaio de compressão, além da diminuição da instabilidade dimensional. Desta forma, a madeira do alburno termorretificada pode tornar-se tecnologicamente mais próxima da madeira do cerne, o que possibilitaria um aumento de rendimento de produtos madeireiros de qualidade.

\section{Referências}

ABNT. Associação Brasileira de Normas técnicas (1997) NBR 7190: Projeto de estruturas de madeiras. São Paulo: ABNT. 107p.

Bachle H, Zimmer B, Windeisen E, Wegener G (2010) Evaluation of thermally modified beech and spruce wood and their properties by FT-NIR spectroscopy. Wood Science Technology, v.44, p.421-433, 2010. doi: 10.1007/s00226-010-0361-3

Calvo-Rodriguez S, Espírito-Santo MM, Nunes YRF, Calvo-Alvarado J (2017) Tree diameter growth for three successional stages of tropical dry forest in Minas Gerais, Brazil. Revista Forestal Mesoamericana Kurú, 14(35): 24-32. doi: 10.18845/rfmk.v14i35.3150

Eleotério JR, Reichert D, Hornburg KF, Meneguelli I (2015) Massa específica e retratibilidade da madeira de seis espécies de Eucalipto cultivadas no litoral de Santa Catarina. Floresta, 45(2): 329-336. doi: 10.5380/rf.v45i2.34699.

Glass SV, Zelinka SL (2010) Moisture relations and physical properties of wood. In: Ross RJ (ed) Wood Handbook, wood as an engineering material. Madison: USDA Forest Service. p. 4.1-4.19. 
Ivanov MMM, Lacerda CF, Paiva FEF, Cavalcante ACR, Oliveira TS (2019) Environmental parameters and tree physiology in land use systems in Brazil. Journal of Forestry Research, 30(2):397-407. doi: 10.1007/s11676-018-0665-y

Kill LHP, Porto DD (2019) Bioma Caatinga: oportunidades e desafios de pesquisa para o desenvolvimento sustentável. In: Vilela, EF, Callegaro, GM, Fernandes, GW (Org.). Biomas e agricultura: oportunidades e desafios. Rio de Janeiro: Academia Brasileira de Ciência FAPEMIG. p. 65-80.

Klitzke RJ, Savioli DL, Muniz GIB, Batista DC (2008) Caracterização dos lenhos de cerne, alburno e transição de jatobá (Hymenae sp.) visando ao agrupamento para fins de secagem convencional. Scientia Forestalis, 36(80):279-284.

Lima JRF, Lima GDS, Lucena CM, Carvalho TKN, Lucena RFP (2018) Inventário in situ como método para avaliação da extração de recursos madeireiros na Caatinga: estudo de caso no município de Cabeceiras (Paraíba, Brasil). Revista Nordestina de Biologia, 26(1):1-18. doi:10.22478/ufpb.22361480v26n1.46051

Maia JH, Ferreira LMM, Castro VG (2020) Influence of extractives on the color of woods from Caatinga. Advances in Forestry Science, 7(2):10431048. doi: 10.34062/afs.v7i2.9421

Melo JE, Camargos JAA (2016) A madeira e seus usos. Brasília: SFB/LPF/MMA. 228p.

Melo JIM, Paulino RC, Oliveira RC, Vieira DD (2018) Flora of Rio Grande do Norte, Brazil: Boraginales. Phytotaxa, 357(4):235-260. doi: 10.11646/phytotaxa.357.4.1

Menezes WM, Souza JT, Carvalho DE, Talgatti M, Santini EJ (2019) Mechanical properties of thermally modified Corymbia citriodora and Eucalyptus saligna woods. Floresta e Ambiente, 26(1):e20150114. doi: 10.1590/2179-8087.011415

Modes KS, Santini EJ, Vivian MA, Haselein CR (2017) Efeito da termorretificação nas propriedades mecânicas das madeiras de Pinus taeda e Eucalyptus grandis. Ciência Florestal, 27(1):291-302. doi: 10.5902/1980509826467

Nogueira SS, Costa JRS, Oliveira AGS, Freitas CBA, Alves AR, Castro VG (2019) Propriedades físicas inter e intra espécies da Caatinga. Ciência da Madeira, 11(1):23-31. doi: 10.12953/21776830/rcm.v11n1p23-31

Roszyk E, Stachowska E, Majka J, Mania P, Broda M (2020) Moisture-dependent strength properties of thermally-modified Fraxinus excelsior wood in compression. Materials, 13(7):1647. doi: 10.3390/ma13071647

Schulz HR, Acosta AP, Barbosa KT, Silva Júnior MAP, Gallio E, Delucis RA, Gatto DA (2021) Chemical, mechanical, thermal, and colorimetric features of the thermally treated Eucalyptus grandis wood planted in Brazil. Journal of the Korean Wood Science and Technology, 49(3):226-233. doi:10.5658/WOOD.2021.49.3.226

Severo ETD, Calonego FW, Sasígolo CA (2012) Physical and chemical changes in juvenile and mature woods of Pinus elliottii var. elliottii by thermal modification. European Journal of Wood Products, 70, p.741-747. doi: 10.1007/s00107-0120611-1

Severo ETD, Calonego FW, Sasígolo CA, Bond B (2016) Changes in the chemical composition and decay resistance of thermally-modified Hevea brasiliensis wood. PLOS ONE, 11(3):e0151353. doi: 10.1371/journal.pone.0151353

Sikora A, Kacik F, Gaff M, Vondrová V, Bubenníková T, Kubovsky I (2019) Impact of thermal modification on color and chemical changes of spruce and oak wood. Journal of Wood Science, 64:406-416. doi: 10.1007/s10086-018-1721-0

Silva JLC, Vidal, CAS, Barros LM, Freita FRV (2018) Aspectos da degradação ambiental no nordeste do Brasil. Revista Gestão \& Sustentabilidade Ambiental, 7(2):180-191. doi: 10.19177/rgsa.v7e22018180-191

Souza GO, Pimenta AS, Trianoski R, Melo RR, Barbosa TK, Castro RVO (2020) Production of edge-glued panels with wood from three species and five types of adhesives. International Wood Products Journal, 11(2):57-63. doi: $10.1080 / 20426445.2020 .1721041$

Tuncer FD, Dogu AD (2018) Effects of heat treatment on some macroscopic and physical properties of Scots pine sapwood and heartwood. Forestist, 68(2):93-100. doi: 10.26650/forestist.2018.34329

Zaque LAM, Melo RR, Souza EC, Serenine Júnior L (2019) Caracterização colorimétrica e da massa específica das madeiras comercializadas no Mato Grosso. Ciência da Madeira, 10(3):236-246. doi: 10.12953/2177-6830/rcm.v10n3p236-246 